\title{
Development of Optimal Kids Insulin Dosing System Formulas for Young Children with Type 1 Diabetes Mellitus
}

\author{
Ramin Alemzadeh, M.D., ${ }^{1,2}$ Raymond G. Hoffmann, Ph.D., ${ }^{3}$ \\ Mahua Dasgupta, M.S., and Elaine Parton, P.N.P.
}

\begin{abstract}
Objective: This study was designed to develop predictive formulas for precise insulin dosing in young children with type 1 diabetes (T1DM).

Research Design and Methods: Consecutive 1-year data from a group of 14 young patients (eight girls, six boys) $3.9 \pm 0.8$ years old with diabetes duration of $2.0 \pm 0.8$ years, transitioned from multiple daily injections (MDI) to continuous subcutaneous insulin infusion (CSII), were analyzed to identify parameters governing optimal insulin dosing. Body mass index (BMI), total daily dose (TDD), total basal dose, insulin-to-carbohydrate ratio (ICR), correction factor (CF), and mean amplitude of glycemic excursion (MAGE) by continuous glucose monitoring and hemoglobin $\mathrm{A}_{1 \mathrm{c}}\left(\mathrm{HbA}_{1 \mathrm{c}}\right)$ level were evaluated at baseline and every 3 months. The slopes of $\mathrm{CF}$ versus $1 / \mathrm{TDD}$, bolus versus TDD, ICR versus $1 / \mathrm{TDD}$, and CF versus ICR were determined.

Results: Kids Insulin Dosing System (KIDS) slope constants at follow-up were associated with MAGE compared with baseline $(P<0.0001)$ without significant changes in BMI $\left(16.6 \pm 1.5\right.$ vs. $\left.16.7 \pm 1.4 \mathrm{~kg} / \mathrm{m}^{2}\right)$ and $\mathrm{HbA}_{1 \mathrm{c}}$ values $(8.0 \pm 0.50 \%$ vs. $7.8 \pm 0.40 \%)$. The relationship between CF and TDD changed significantly during CSII compared with baseline MDI $(P<0.0001)$, whereas the coefficients for ICR and TDD relationship remained relatively unchanged. The KIDS formulas estimated TDD $=0.74 \times$ body weight, total basal dose $=0.28 \times \mathrm{TDD}, \mathrm{CF}=2,800$ / TDD, and ICR $=13.5 \times$ body weight $/$ TDD.

Conclusions: The interrelationships among ICR, CF, TBD, and TDD remained stable on CSII and were accompanied by decreased glycemic excursions. The KIDS formulas may yield consistent and easy estimates of insulin dosing factors in very young patients with T1DM.
\end{abstract}

\section{Introduction}

$\mathbf{P}$ ATIENTS With DiABETES on basal bolus insulin regimen using either multiple daily injections (MDI) or continuous subcutaneous insulin infusion (CSII) must know both carbohydrate meal content and premeal blood glucose (BG) values for precise insulin dosing. The carbohydrate content of a meal is divided by an insulin sensitivity factor, also known as the insulin-to-carbohydrate ratio (ICR). The current formulas for estimation of ICR range from 300 to 450 divided by the total daily dose (TDD) or from 4.1 to 6.2 times the weight (in $\mathrm{kg}$ ) divided by the TDD. ${ }^{1-3}$ For instance, in a patient with an ICR of 1.0 unit/15 g, consuming $75 \mathrm{~g}$ of carbohydrate will require 5 units of insulin ( $75 / 15=5.0$ units). In addition, if the premeal BG exceeds the target, the difference is divided by another insulin sensitivity factor, known as the correction factor $(\mathrm{CF})$. The current recommendation for estimating $\mathrm{CF}$ is
1,500 or 1,700 divided by the TDD. For instance, if the BG is $120 \mathrm{mg} / \mathrm{dL}$ higher than target and the CF is $40 \mathrm{mg} / \mathrm{dL} /$ unit of insulin, then 3 units of additional insulin is administered $(120 / 40=3$ units).

The formulas for estimating ICR and CF were derived from retrospective and prospective observations of adult type 1 diabetes mellitus (T1DM) patients treated with CSII. ${ }^{1-3}$ It has been shown that hemoglobin $\mathrm{A}_{1 \mathrm{c}}\left(\mathrm{Hb}_{1 \mathrm{c}}\right)$ does not correspond to daily BG variability evaluated by continuous glucose monitoring (CGM). ${ }^{4-6}$ Indeed, BG excursions as measured by mean amplitude of glycemic excursion (MAGE) were found to correlate with bolus:basal insulin ratio and hypoglycemic events independent of age, mean BG, and glycemic control. ${ }^{7,8}$

Although daily glucose trend analyses and treatment adjustment using CGM have been used to derive insulin dose estimation formulas in adults with $\mathrm{T} 1 \mathrm{DM},{ }^{9,10}$ there are

\footnotetext{
${ }^{1}$ Department of Pediatrics, Medical College of Wisconsin, Milwaukee, Wisconsin.

${ }^{2}$ University of Illinois at Chicago, Chicago, Illinois.

${ }^{3}$ Children's Research Institute, Milwaukee, Wisconsin.
} 
currently no optimal dosing guidelines for young children with T1DM. Therefore, the aim of this study was to derive formulas for estimation of optimal basal insulin, ICR, and CF in young children with T1DM who had undergone CGM evaluation during CSII over a 1-year period. ${ }^{11}$ We designated these formulas as Kids Insulin Dosing System (KIDS).

\section{Subjects and Methods}

Fourteen children (eight girls, six boys) 2.2-5.5 years old with a duration of diabetes of 1.0-3.3 years were recruited from the Children's Hospital of Wisconsin, Milwaukee, WI, for a 1-year prospective study as previously published. ${ }^{11}$ Parents/guardians were required to provide constant supervision and monitor BG levels at least four times a day. Prior to initiation of CSII, all patients received insulin MDI consisting of mealtime aspart (Novolog ${ }^{\circledR}$, Novo Nordisk Pharmaceuticals, Inc., Princeton, NJ) and bedtime glargine (Lantus ${ }^{\circledR}$, Sanofi-Aventis Pharmaceuticals, Inc., Bridgewater, NJ) insulin and applied principles of mealtime carbohydrate adjustment using ICR and CF. Patients were evaluated every 3 months during visits to the Diabetes Clinic.

Baseline and 1-year prospective data were collected on CSII therapy using meter download logbooks and the CGM system. At each clinic visit, height, weight, body mass index (BMI), and $\mathrm{HbA}_{1 \mathrm{c}}$ were obtained, and the number of moderate $(\mathrm{BG}<60 \mathrm{mg} / \mathrm{dL}[<3.3 \mathrm{mmol} / \mathrm{L}]$ with or without behavioral impairment) and severe (BG $<50 \mathrm{mg} / \mathrm{dL}[<2.8 \mathrm{mmol} / \mathrm{L}]$ associated with unconsciousness with or without seizure) hypoglycemic episodes in the preceding 3 months and during the study was determined as previously reported. ${ }^{11}$ This study was approved by the Children's Hospital of Wisconsin Institutional Review Board, and informed consent for participation in the study was obtained from parents/guardians of patients.

\section{Insulin dosage calculations and $H b A_{1 c}$ determination}

All patients were started on a Medtronic/MiniMed (Northridge, CA) insulin pump, and parents/guardians received extensive training on the use of pump use and its risks, including catheter site infection, hyperglycemia, hypoglycemia, ketosis and diabetic ketoacidosis, and potential mechanical problems (i.e., kinked infusion sets, air bubbles, and dislodged tubing).

Aspart insulin was used in all pumps. Baseline TDD for CSII was calculated from the 1-week average of the previous TDD (aspart+glargine regimen). The insulin glargine dose was used for basal rates, and the remainder was used for bolus dosing. An initial insulin dosage algorithm was individualized in most patients during the year to accommodate variable insulin sensitivity. The premeal BG target was established at $80-150 \mathrm{mg} / \mathrm{dL}$ at $2 \mathrm{~h}$ postprandial with the target glucose (TG) of $180 \mathrm{mg} / \mathrm{dL}$.

$\mathrm{HbA}_{1 \mathrm{c}}$ was determined using the Bayer DCA 2000 instrument (Bayer Diagnostics Inc., Tarrytown, NY), with a range in those without diabetes of $4.5-5.7 \%$.

\section{CGM system}

Baseline and quarterly CGM system (MiniMed CGMS ${ }^{\circledR}$ System Gold) tracings were obtained every 3 months for 1 year in each patient. Patients performed at least four self- monitoring of BG (SMBG) tests per day and entered these values into the CGMS monitor to obtain correlation coefficients between the SMBG and the CGMS values. All patients/ guardians were blinded to the CGMS data. The CGMS tracings were deemed accurate if (1) the correlation coefficient between the paired sensor and meter readings was above the cutoff point of 0.79 and (2) the mean absolute difference of sensor and meter readings was below $28 \%$.

In addition, detailed accounts of time and quantity of carbohydrate contents of food and insulin doses, as well as symptoms and duration of hypoglycemic events, were recorded by families. The CGMS tracings ranged from 5 to 6 days, and mean BG (MBG) and MAGE were used to assess BG variability in patients with T1DM as previously described. ${ }^{12,13}$

\section{Statistical analysis}

Our primary outcome was an observed percentage change in $\mathrm{HbA}_{1 \mathrm{c}}$. With $13 \mathrm{~T} 1 \mathrm{DM}$ children, we had $80 \%$ power to detect a difference of $0.3(\alpha=0.05)$ in $\mathrm{HbA}_{1 \mathrm{c}}$ between baseline and 1.0year follow-up. ${ }^{14}$ The reported values represent the mean \pm SD. Baseline and 1.0-year follow-up characteristics were compared with paired $t$ test and $\chi^{2}$ analyses, and when differences were found, they were controlled for in further analyses. The $\mathrm{HbA}_{1 \mathrm{c}}$ data were analyzed using a paired-samples $t$ test. The number of MBG, MAGE, and frequency and duration of hypoglycemic events were analyzed using a repeated-measures analysis of variance with a test for trend over time.

The slopes of the CF versus 1/TDD, bolus versus TDD, ICR versus $1 / \mathrm{TDD}$, and $\mathrm{CF}$ versus ICR were determined. Linear and nonlinear least squares were used to fit the models. The models for all five time points (baseline and 3, 6, 9, and 12 months) were assessed separately. However, the models at 6 months and at 12 months were not significantly different. Hence, a longitudinal error structure with an autoregressive model for the repeated measures at 6 months and 12 months was used to increase precision. This was used to combine data from the two time points ( 6 and 12 months). A linear model was created for drawing the nomogram. ${ }^{15}$ No intercept models (autoregressive error structure) were created to determine the quick formulas for some important measures. Analysis of variance was used to test the difference of the slopes and other variables. Using an autoregressive model for repeated measures at 6 and 12 months, a linear model for drawing the nomogram was created. $P<0.05$ was considered significant.

\section{Results}

Clinical and biochemical characteristics of 14 children (eight girls, six boys) with T1DM at baseline, 6 months, and 1 year after initiation of CSII are shown in Table 1. BMI, MBG, $\mathrm{HbA}_{1 \mathrm{c}}$ and number and mean of hypoglycemic events were not significantly affected after 1 year of CSII, whereas $\mathrm{HbA}_{1 c}$ was marginally decreased. However, MAGE values were significantly lower at the 6- and 12-month follow-up visits compared with baseline values despite similar number of BG excursions $(P<0.0001)$.

Table 2 summarizes mean TDD, bolus and basal insulin between TDD, ICR, and CF at baseline, 6 months, and 1 year after initiation of CSII. Table 2 also displays the interrelationships of TDD and ICR with CF and bolus and basal insulin. The coefficients of the hyperbolic equations are shown; 
Table 1. Clinical and Biochemical Profiles at Baseline, 6 Months, and 12 Months

\begin{tabular}{|c|c|c|c|c|}
\hline Parameter & Baseline NTG & 6 month TG & 12 month TG & $\mathrm{P}$ \\
\hline Age (years) & $3.9 \pm 0.8^{\mathrm{a}}$ & $4.4 \pm 0.8$ & $4.9 \pm 0.6$ & $<0.003$ \\
\hline BMI $\left(\mathrm{kg} / \mathrm{m}^{2}\right)$ & $16.5 \pm 1.5$ & $17.0 \pm 1.3$ & $16.7 \pm 1.4$ & NS \\
\hline $\mathrm{MBG}(\mathrm{mg} / \mathrm{dL})$ & $213 \pm 94$ & $207 \pm 81$ & $185 \pm 79$ & NS \\
\hline Number of hypoglycemic events & $2.8 \pm 1.8$ & $3.6 \pm 2.8$ & $2.8 \pm 2.5$ & NS \\
\hline Mean hypoglycemic values (mg/dL) & $52 \pm 5$ & $51 \pm 3$ & $52 \pm 4$ & NS \\
\hline Nocturnal hypoglycemia (min) & $135 \pm 141$ & $139 \pm 107$ & $120 \pm 103$ & NS \\
\hline Total hypoglycemia (min) & $267 \pm 222$ & $205 \pm 122$ & $189 \pm 148$ & NS \\
\hline Number of BG excursions & $17.1 \pm 4.1$ & $17.2 \pm 4.8$ & $15.7 \pm 3.0$ & NS \\
\hline MAGE $(\mathrm{mg} / \mathrm{dL})$ & $210 \pm 31^{b}$ & $176 \pm 23$ & $168 \pm 22$ & $<0.0001$ \\
\hline $\mathrm{HbA}_{1 \mathrm{c}}(\%)$ & $8.0 \pm 0.50$ & $7.7 \pm 0.4$ & $7.8 \pm 0.40$ & 0.068 \\
\hline
\end{tabular}

Data are mean $\pm \mathrm{SD}$ values.

${ }^{\mathrm{a}} P<0.003,{ }^{\mathrm{b}} P<0.0001$, baseline versus 6 and 12 months.

$\mathrm{BG}$, blood glucose; $\mathrm{BMI}$, body mass index; $\mathrm{HbA}_{1 c}$, hemoglobin $\mathrm{A}_{1 \mathrm{c}}$; MAGE, mean amplitude of glycemic excursion; MBG, mean blood glucose; NS, not significant; NTG, non-target glucose; TG, target glucose.

these were used to obtain the quick formulas, which essentially stabilized at 6 months. The observed and predicted CF values changed significantly at 6 and 12 months compared with baseline $(P<0.001)$, as did the bolus insulin and TDD $(P=0.03)$. The $R^{2}$ (e.g., 0.97 or $97 \%$ for CF vs. $1 /$ TDD) shows how well the equation describes the data (the maximum possible is $100 \%$ for a perfect fit). CF was independent of body weight. On the other hand, the coefficients for the ICR and TDD and the CF and ICR relationships remained relatively unchanged at the three time points. In addition, the observed $C F$ values showed individual variability independent of body weight $(P=0.42)$.

Figure 1 is the nomogram that was developed from 6- and 12-month data. This three-line nomogram consists of CF/ TDD, ICR, and body weight parallel lines from left to right, respectively. The dotted line is a sample patient with body weight $=17.2 \mathrm{~kg}, \mathrm{TDD}=11.5$ units, $\mathrm{ICR}=1$ unit $/ 22 \mathrm{~g}$, and $\mathrm{CF}=1 \mathrm{unit} / 250 \mathrm{mg} / \mathrm{dL}$.

The exact computations for the nomogram were developed based on the 6- and 12-month data and included the body weight using a log transform of the hyperbolic model, which was then transformed back to the original coordinates. Total daily insulin or TDD was estimated at $0.74 \mathrm{U} \times$ body weight with basal dose of $0.28 \mathrm{U} \times \mathrm{TDD}$. The ICR model was esti- mated as ICR $=28.9+(0.43 \times$ body weight $)-(1.24 \times \mathrm{TDD})$ with the ICR quick formula $=13.5 \times$ body weight $/$ TDD. The $\mathrm{CF}$ was estimated as $\mathrm{CF}=2,841.2$ /TDD with the quick $\mathrm{CF}$ formula $=2,800 /$ TDD.

\section{Discussion}

In our study, interrelationships among ICR, CF, basal insulin, and TDD remained stable on CSII and were accompanied by decreased BG excursions. This study included very young normal weight children with T1DM. These predicted formulas for estimation of insulin dosing parameters may be generalized to preschool-aged children.

In this study, insulin pump therapy decreased glycemic instability without further improving $\mathrm{HbA}_{1 \mathrm{c}}$ or altering frequency and duration of hypoglycemic events in a group of young children with T1DM as previously published. ${ }^{11}$ Lack of glycemic improvement and reduction in hypoglycemia in our patients may suggest significant diabetes management challenges in young patients as it relates to variable levels of physical activity, food intake, and changing insulin sensitivity in a growing child. ${ }^{16}$ It may also suggest that families could not maintain the same level of interest despite frequent contacts with the diabetes team for education and support

Table 2. Interrelationships Among Total Daily Dose, Bolus Insulin, Basal Insulin, InSUlin-TO-CARbohydRATE RATIO, AND CORRECTION FACTOR

\begin{tabular}{|c|c|c|c|c|}
\hline Parameter & Baseline NTG & 6 month TG & 12 month TG & $\mathrm{P}$ \\
\hline TDD (U/day) & $13.3 \pm 4.0^{\mathrm{b}}$ & $14.4 \pm 3.6$ & $15.6 \pm 3.7$ & $<0.05$ \\
\hline Bolus (U/day) & $8.7 \pm 2.7^{b}$ & $10.1 \pm 3.2$ & $10.9 \pm 2.6$ & $<0.05$ \\
\hline Basal (U/day) & $4.6 \pm 1.9$ & $4.3 \pm 1.3$ & $4.8 \pm 1.4$ & NS \\
\hline Basal insulin (\%) & $33.6 \pm 7.1$ & $30.6 \pm 8.9$ & $30.4 \pm 5.1$ & NS \\
\hline CF vs. TDD & $2,223 / \mathrm{TDD}^{\mathrm{a}}$ & $2,860 / \mathrm{TDD}$ & 2,830/TDD & $<0.0001$ \\
\hline$R^{2}$ for CF vs. $1 / \mathrm{TDD}$ & 0.980 & 0.988 & 0.985 & NS \\
\hline$R^{2}$ for bolus vs. TDD & $0.65^{b}$ & 0.71 & 0.70 & $<0.05$ \\
\hline ICR & $12.7 \times \mathrm{BW} / \mathrm{TDD}$ & $13.3 \times \mathrm{BW} / \mathrm{TDD}$ & $13.7 \times \mathrm{BW} / \mathrm{TDD}$ & NS \\
\hline$R^{2}$ for ICR vs. $1 / \mathrm{TDD}$ & 0.986 & 0.993 & 0.991 & NS \\
\hline$R^{2}$ for CF vs. ICR & 9.48 & 10.65 & 9.79 & NS \\
\hline
\end{tabular}

Data are mean \pm SD values.

${ }^{\mathrm{a}} P<0.0001,{ }^{\mathrm{b}} P<0.05$, baseline versus 6 and 12 months.

BW, body weight; CF, correction factor; ICR, insulin-to-carbohydrate ratio; NS, not significant; NTG, non-target glucose; TDD, total daily dose; TG, target glucose. 


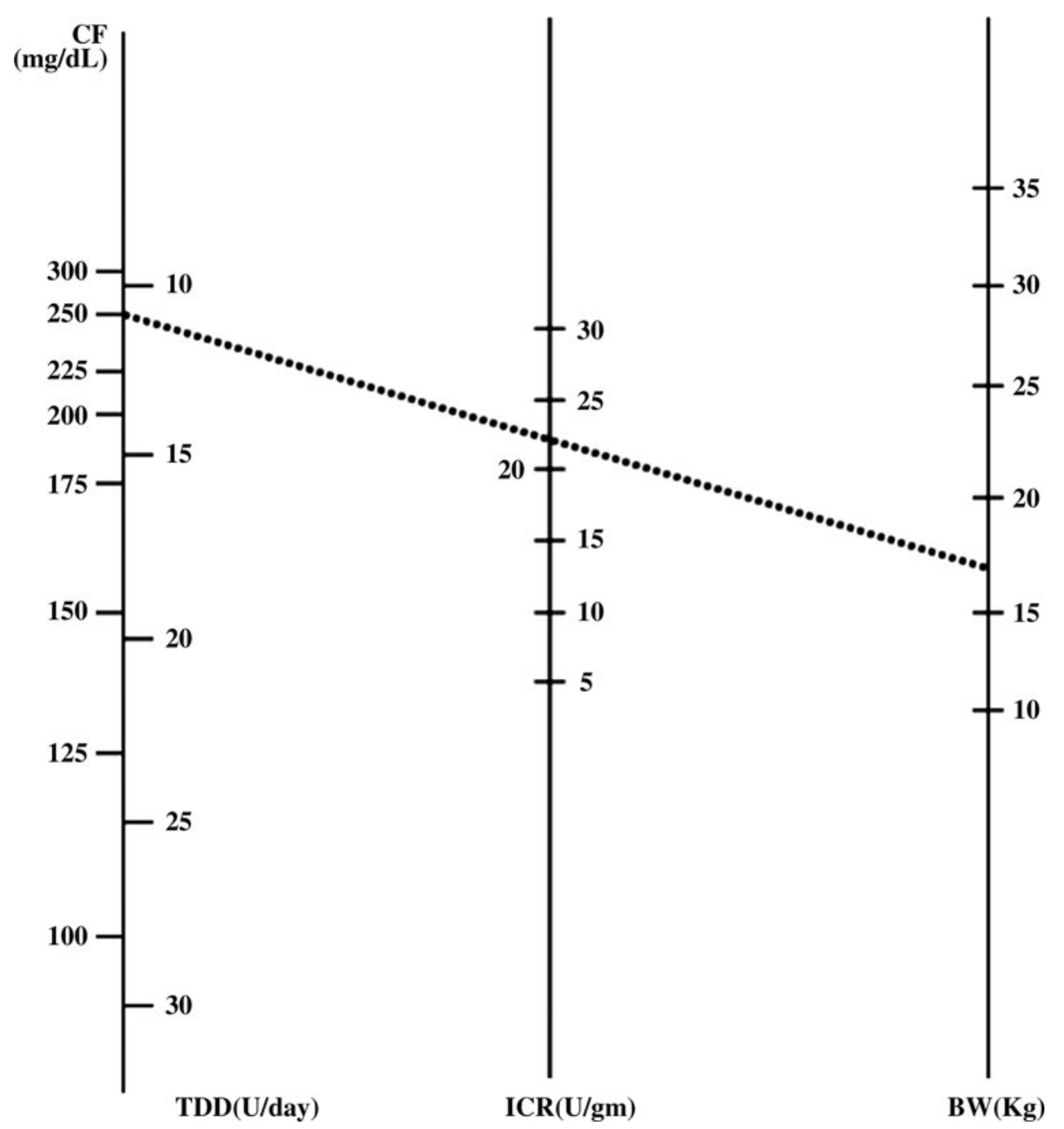

FIG. 1. Nomogram plot. A sample patient is shown by the dotted line. BW, body weight; CF, correction factor; ICR, insulinto-carbohydrate ratio; TDD, total daily dose.

throughout the study. However, we observed significant reduction in glucose variability as expressed by MAGE as previously shown. ${ }^{17}$

During insulin pump therapy, the basal and bolus dosing factors remained stable except for comparison with baseline. This suggests that improved BG stability resulted in improved estimation of insulin sensitivity. In our study, ICR and CF dosing formulas derived from patient data are markedly different from those reported previously in well-controlled T1DM adults treated with CSII. ${ }^{1-4}$ This is the first study attempting to estimate insulin dosing formulas in young children using CGM. Although the glycemic control as measured by $\mathrm{HbA}_{1 \mathrm{c}}$ was not significantly improved, reduction in $\mathrm{BG}$ variability allowed for consistent estimation of ICR and CF formulas in this prospective study. However, we were not able to achieve consistently target postmeal BG levels, which may explain absence of improved glycemic control. There was no significant difference between ICR and CF between mealtimes as previously shown, ${ }^{18}$ whereas some have suggested higher bolus insulin requirement especially in the morning due to the dawn phenomenon ${ }^{19}$ or inadequate glucose control prior to the meal. ${ }^{20}$
In our cohort, we found strong relationships among 1/ TDD, basal dose, ICR, and CF. Therefore, any adjustment in any insulin dosing factor may require modification in other factors. Indeed, these findings were similar to that found in a study by King and Armstrong ${ }^{2}$ that derived insulin dosing formulas from a 2-week prospective study in T1DM adults. The vast differences between KIDS formulas and those used in older patients are primarily due to lower BMI but also higher insulin sensitivity in children.

The limitations of the study included (1) the small sample of children, (2) exclusion of pubertal children, and (3) use of standard CGM instead of real-time CGM. However, we used insulin dose data from repeated visits and evaluated inbetween meals SMBG data for postmeal glucose adjustments. In addition, development of insulin dosing formulas is a greatly needed tool due to the rising incidence of T1DM in very young children ${ }^{21}$ and their unique insulin requirement in order to minimize BG instability and hypoglycemic events. Finally, the use of the KIDS nomogram can save time, increase accuracy, help patients/guardians understand relationships between various dosing factors, and help teach other clinicians. 
In conclusion, our data support specific insulin dosing formulas for young children with T1DM. The KIDS formulas can yield consistent and easy estimates of insulin dosing factors in very young patients with T1DM. Nevertheless, insulin dosing requires clinical judgment and optimal BG monitoring because the formulas may vary widely from the observed findings.

\section{Acknowledgments}

The previous study cited by Alemzadeh et al. ${ }^{11}$ was funded by NovoNordisk Pharmaceuticals, Inc. and Medtronic, Inc.

\section{Author Disclosure Statement}

R.A. researched data and wrote the manuscript, R.H. helped design and performed the study design biostatistical analyses and contributed to the manuscript, M.D. researched data and performed statistical analysis, and E.P. researched data and wrote and edited the manuscript. No competing financial interests exist.

\section{References}

1. Davidson PC, Hebblewhite HR, Steed RD, Bode BW: Analysis of guidelines for basal-bolus insulin dosing: basal insulin, correction factor and carbohydrate-to-insulin ratio. Endocr Pract 2008;14:1095-1101.

2. King $A B$, Armstrong DU: A prospective evaluation of insulin dosing recommendations in patients with type 1 diabetes at near normal glucose control: bolus dosing. J Diabetes Sci Technol 2007;1:42-46.

3. King AB: How much do I give? Revaluation of insulin dosing estimation formulas using continuous glucose monitoring. Endocr Pract 2010;16:428-432.

4. Gross TM, Mastrototaro JJ: Efficacy and reliability of the continuous glucose monitoring system. Diabetes Technol Ther 2000;2(Suppl 1):S-19-S-26.

5. Ludvigsson J, Hanas R: Continuous subcutaneous glucose monitoring improved metabolic control in pediatric patients with type 1 diabetes: a controlled crossover study. Pediatrics 2003;111:933-938.

6. Kaufman FR, Gibson LC, Halvorson M, Carpenter S, Fisher LK, Pitukcheewanont P: A pilot study of the continuous glucose monitoring system: clinical decisions and glycemic control after its use in pediatric type 1 diabetic subjects. Diabetes Care 2001;24:2030-2034.

7. Alemzadeh R, Loppnow C, Parton E, Kirby M: Glucose sensor evaluation of glycemic instability in pediatric type 1 diabetes mellitus. Diabetes Technol Ther 2003;5:167-173.

8. Alemzadeh R, Palma-Sisto P, Parton EA, Holzum MK: Continuous subcutaneous insulin infusion and multiple dose of insulin regimen display similar patterns of blood glucose excursions in pediatric type 1 diabetes. Diabetes Technol Ther 2005;7:587-596.

9. King $\mathrm{AB}$, Armstrong DU: Daily glucose trend analyses and treatment adjustments using the continuous glucose monitoring system (CGMS, Medtronic MiniMed): "tweaking." Diabetes Technol Ther 2004;6:297-298.
10. King $A B$, Armstrong DU: A prospective evaluation of insulin dosing recommendations in patients with type 1 diabetes at near normal glucose control: basal dosing. J Diabetes Sci Technol 2007;1:36-41.

11. Alemzadeh R, Palma-Sisto P, Holzum M, Parton E, Kichler J: Continuous subcutaneous insulin infusion attenuated glycemic instability in preschool children with type 1 diabetes mellitus. Diabetes Technol Ther 2007;9:339-347.

12. Service FJ, Molnar GD, Rosevear JW, Ackerman E, Gatewood LC, Taylor WF: Mean amplitude of glycemic excursions, a measure of diabetic instability. Diabetes 1970;19: 644-655.

13. Molnar GD, Taylor WF, Ho MM: Day-to-day variation of continuously monitored glycemia: a further measure of diabetic instability. Diabetologia 1972;8:342-348.

14. Boland E, Grey M, Osterle A, Fredrickson L, Tamborlane W: Continuous subcutaneous insulin infusion: a new way to lower risk of severe hypoglycemia, improve metabolic control, and enhance coping in adolescents with type 1 diabetes. Diabetes Care 1999;22:1779-1784.

15. Brodetsky S: A First Course in Nomography. London: G. Bell and Sons, Ltd., 1920.

16. Danne $T$, von Schütz W, Lange $K$, Nestoris C, Datz N, Kordonouri O: Current practice of insulin pump therapy in children and adolescents-the Hannover recipe. Pediatr Diabetes 2006;7(Suppl 4):25-31.

17. Jeha GS, Karaviti LP, Anderson B, Smith EO, Donaldson S, McGirk TS, Haymond MW: Insulin pump therapy in preschool children with type 1 diabetes mellitus improves glycemic control and decreases glucose excursions and the risk of hypoglycemia. Diabetes Technol Ther 2005;7:876-884.

18. Bode BW: Insulin pump therapy. In: Lebovitz $\mathrm{H}$, ed. Therapy for Diabetes Mellitus and Related Disorders, $4^{\text {th }}$ ed. Alexandria, VA: American Diabetes Association, 2004:224231.

19. Scheiner G, Boyer BA: Characteristics of basal insulin requirements by age and gender in Type- 1 diabetes patients using insulin pump therapy. Diabetes Res Clin Pract 2005; 69:14-21.

20. Service FJ, Rizza RA, Hall LD, Westland RI, O'Brien PC, Clemens AH, Haymond MW, Gerich JE: Prandial insulin requirements in insulin-dependent diabetics: effects of size, time of day and sequence of meals. J Clin Endocrinol Metab 1983;57:931-936.

21. Evertsen J, Alemzadeh R, Wang X: Increasing incidence of pediatric type 1 diabetes mellitus in Southeastern Wisconsin: relationship with body weight at diagnosis. PLoS One 2009;4:e6873.

Address correspondence to: Ramin Alemzadeh, M.D. Professor of Pediatrics Chief, Division of Pediatric Endocrinology E Diabetes University of Illinois at Chicago 840 South Wood Street, M/C 856, 1330 CSB Chicago, IL 60612

E-mail: ralemzad@uic.edu 